\title{
Estrategias didácticas en los procesos de enseñanza de la educación física escolar mediadas por las TICS durante el confinamiento por COVID 19
}

Didactic strategies in the teaching processes of school physical education mediated by ICTs during confinement by COVID 19

\author{
John Edwin Valencia Moreno ${ }^{1}$ \\ Universidad del Magdalena
}

Recibido: 28.08.2021

Aceptado: 30.10 .2021

\section{Resumen}

El presente estudio tiene como propósito describir e interpretar las estrategias didácticas de la educación física escolar mediadas por las TICS durante el confinamiento por Covid 19. La investigación se enmarca en el paradigma de la investigación cualitativa con corte interpretativo-descriptivo y diseño metodológico etnográfico educativo. En cuanto a las técnicas para la recolección de datos, se realizaron observaciones de clase en cuatro colegios distritales de la ciudad de Bogotá. Los resultados evidencian una tendencia de clases sincrónicas a través del uso de las plataformas Teem, Meet, asimismo, el WhatsApp y correo electrónico como las herramientas didácticas que usan los docentes para desarrollar sus procesos de enseñanza durante el confinamiento. Con respecto a las estrategias didácticas, se resaltan los ejercicios guiados y la imitación. Por otra parte, se describe el desarrollo de la condición física la cual hace parte de la competencia motriz. Conclusiones: Se evidencia la

\footnotetext{
1 johnvalenciaem@unimagdalena.edu.co https://orcid.org/0000-0002-2168-9068 
necesidad de formación docente sobre las herramientas didácticas tecnológicas, para facilitar la implementación de estrategias didácticas, que permitan desarrollar las competencias de la educación física en clases no presenciales.

Palabras clave: Educación física; Tecnologías de la información y comunicación; Didáctica; Educación.

\begin{abstract}
The present study aims to describe and interpret the didactic strategies of school physical education mediated by ICTs during confinement by Covid 19. The research is framed within the paradigm of qualitative research with an interpretative-descriptive cut and an educational ethnographic methodological design. Regarding the techniques for data collection, class observations were made in four district schools in the city of Bogotá. The results show a trend of synchronous classes through the use of the platforms Teem, Meet, likewise, WhatsApp and email as the didactic tools that teachers use to develop their teaching processes during confinement. With regard to teaching strategies, guided exercises and imitation are highlighted. On the other hand, the development of physical condition is described, which is part of motor competence. Conclusions: The need for teacher training on technological didactic tools is evidenced, to facilitate the implementation of didactic strategies, which allow the development of physical education competencies in non-contact classes.
\end{abstract}

Keywords: Physical education; Information and communication technologies; Teaching for understanding; Education 


\section{Introducción}

El área de educación física en Colombia hace parte de las áreas obligatorias y fundamentales de la ley general de educación del año 1994, ARTICULO 23. El objetivo del área es desarrollar la competencia motriz, axiológico corporal, expresivo corporal y las específicas en relación con las básicas, como las ciencias sociales, naturales, ciudanía, matemáticas y comunicativas (Ministerio de Educación Nacional, 2010). En este sentido, los procesos de enseñanza y aprendizaje se pueden abordar desde diferentes estrategias y herramientas didácticas que favorezcan la comprensión en el escolar, y de esta manera el desarrollo de las competencias mencionadas. Según Perkins (1999) "La comprensión es la habilidad para pensar y actuar, creativa y flexiblemente a partir de lo que sabemos, para resolver problemas, crear productos e interactuar con el mundo que nos rodea" (p. 4). Desde esta perspectiva, la enseñanza debe promover el uso de los conocimientos y habilidades en la vida cotidiana de los escolares (Stone, 1999) En otras palabras, las competencias generan dominio de contenidos y habilidades motrices para interactuar en diferentes escenarios de la cotidianidad.

Cabe señalar que, el desarrollo de las competencias se orienta mediante procesos de enseñanza y aprendizaje, los cuales históricamente han sido objetivos de investigación constante. En este contexto, la didáctica emerge como una disciplina encargada estudiar las estrategias de enseñanza, acción pedagógica, estudio y diseño del currículo (Limas, 2000). La didáctica genera diferentes alternativas de enseñanza para facilitar procesos de aprendizaje en los estudiantes. Por otra parte, esta disciplina promueve la interacción con los estudiantes, además de la constante reflexión docente para identificar los intereses y problemas del aula interpretando cuáles son las actividades, estrategias y herramientas, que puedan logran interiorizar los contenidos de forma más significativa (Medina et al. 2009). Asimismo, según Diaz (2009) "La enseñanza de la didáctica suele convertir la complejidad de esta disciplina en un problema simple y superficial. La didáctica no se enseña, en general, solo como un conjunto de estrategias docentes (...) con la finalidad de que el profesor pueda aplicarlas en el escenario escolar" (p.25). 
En consonancia con lo anterior, De camelloni (2008) manifiesta que la didáctica es una disciplina soportada en la enseñanza, por lo cual, estudia el currículo y las estrategias de enseñanza. Para ser más específicos, la enseñanza es el objeto de estudio de los didactas, y ha sido influenciada por las transformaciones políticas, sociales, y pedagógicas de la historia. En la actualidad, encontramos cambios sustanciales en las herramientas didácticas como consecuencia del confinamiento por el virus del Covid 19. En particular, hallamos en esta problemática mundial, el uso de herramientas que poco eran incluidas en los procesos de enseñanza y aprendizaje escolar, como, por ejemplo, las tecnologías de la información y comunicación (TICS). En este panorama, encontramos las TICS como la herramienta didáctica alternativa, que puede facilitar el desarrollo de las competencias de la educación física. Se reportan algunos sobre la implementación de las TICS en la educación física antes del confinamiento, así, por ejemplo, Úbeda y Molina (2016) a través de una experiencia innovadora hicieron uso del blog como herramienta didáctica. Los autores consideran que, el uso del blog permite el acceso a la información y el abordaje de contenidos teóricos sin interrumpir el tiempo dedicado a la actividad motriz. No obstante, Escobar et, al. (2018) hallaron poca tendencia, en cuanto el uso y aprovechamiento de la tecnología en el contexto educativo escolar.

En la actualidad, el uso de las Tics pasó de ser una herramienta para abordar prácticas, investigaciones de experiencias exitosas, y estrategias didácticas con baja tendencia a una necesidad educativa, como consecuencia del confinamiento por el virus Covid 19.Sin embargo, según Kamoga y Varea (2021) Suecia no implemento mediadas de bloqueo, pero el actual escenario generó cambios sustanciales en los maestros, transformaciones en el contexto, roles, contenido, igualmente, los problemas en las estrategias de enseñanza, el contacto físico, la interacción entre estudiantes y maestros. Los autores concluyen en su estudio, que la pandemia genera desafíos educativos, además de crear oportunidades de discusiones, ajustes y posibles escenarios para superar la situación actual en la escuela. Desde esta perspectiva, establecer políticas de formación docente en el uso de las TICS pueden mejorar el paradigma técnico imperante emergiendo modelos alternativos en los procesos de enseñanza y aprendizajes mediados por las TICS (Barahona et al, 2020). 
Es importante destacar que, este virus se origina en la ciudad de Wuhan, en China. Posteriormente, se expande por el planeta, el 12 de marzo la Organización Mundial de la Salud la declara pandemia, y en consecuencia se genera un confinamiento mundial (Ciotti et al,2020). Según la UNESCO (2021) al 30 de junio del 2021 han sido perjudicados 156,692,641 estudiantes. Sin embargo, después de un año de emergencia sanitaria, casi la mitad de los estudiantes del mundo continúan afectados por el cierre total o parcial de las instituciones educativas. El anterior panorama generó cambios en los contextos educativos, entre estos la implementación de las TICS como la principal herramienta para realizar reuniones entre docentes, administrativos, administrativos y docentes, directivos con docentes, padres de familia, en síntesis, toda la comunidad educativa. En este aspecto, los procesos de enseñanza y aprendizaje escolar se desarrollan a través de dispositivos electrónicos como el computador, Tablet o celulares a través del internet.

Con respecto a los resultados del presente estudio, se hallan los procesos de enseñanza con énfasis en la competencia motriz a través de la condición física utilizando estrategias didácticas como la imitación y ejercicios guiados. En cuanto a las herramientas didácticas mediadas por las TICS, se encuentran las clases sincrónicas como la herramienta que permite una interacción más directa entre estudiantes y docente. Según Cañizares et al (2021) para los docentes las clases sincrónicas se presentan como un reto, porque se necesitan de la creatividad, actitud y motivación en el desarrollo de las actividades virtuales con el objetivo de guiar a los estudiantes hacia un trabajo autónomo para llegar la significancia. Sin embargo, e, las clases sincrónicas poco evidenciaron retroalimentación, evaluación continua o relación de las actividades con la vida cotidiana. Otras herramientas diádicas usadas fueron las clases asincrónicas y remotas por medio de guías. Desde esta perspectiva es importante realizar más estudios sobre educación física mediadas por las Tics, ya que, es un campo poco explorado, y de esta manera generar más teoría sobre el desarrollo de las competencias del área a través de las herramientas didácticas TICS. 


\section{Metodología}

La presente investigación se ubica en el paradigma de la investigación cualitativa con corte interpretativo-descriptivo, Según Ortiz (2013) "El paradigma cualitativo reconoce la diversidad y pluralidad de escenarios, condiciones y situaciones que se presentan en la realidad, por lo tanto, son únicas e irrepetibles, así las observaciones y resultados sólo son válidas para el caso particular" (p. 9). En cuanto al diseño metodológico, se aborda la etnografía educativa, pues según Maturana y Garzón (2015) los estudios etnográficos en el contexto educativo permiten estudiar los procesos diádicos, la practica pedagógica y los escenarios educativos. La etnografía no tiene un solo objetivo, sino varios estrechamente asociados, entre estos: "la descripción de los contextos, la interpretación de estos para llegar a su comprensión, la difusión de los hallazgos, y la mejora de la realidad educativa" (Alvares, 2008 p.3)

Con respecto a la unidad de análisis se seleccionan cuatro colegios oficiales de Bogotá, dos de la localidad de Bosa y dos de otras localidades que contaran con básica primaria. Asimismo, se seleccionaron cuatro docentes, uno por cada institución educativa seleccionada, con título de licenciatura en educación física o afines, y nombramiento en propiedad. Por otra parte, se eligieron los grados superiores de la básica primaria, grados quinto y cuarto. Es importante señalar que, la localidad de Bosa cuenta con 28 colegios oficiales y 31 docentes de educación física en la básica primaria nombrados en propiedad (SED,2018). En cuanto a las técnicas para la recolección de datos, se realizaron observaciones de clases a través grabaciones de clases sincrónicas. Las grabaciones permiten capturar las estrategias didácticas que utiliza el docente durante sus clases, la pertinencia de las TICS en los procesos de enseñanza y aprendizaje, las interacciones de los escolares durante las clases, interacción docenteestudiante y estudiante-estudiante. Ahora bien, las observaciones se pueden realizar de diversas formas, entre estas la participación pasiva, y, por otra parte, la participación en la cual el investigador interactúa activamente con los participantes, inclusive volviéndose parte del grupo social. (Peralta, 2009). 
A continuación, se expone el procedimiento realizado en el presente estudio. En primera instancia, se preseleccionaron 6 colegios, 6 docentes de los cuales uno no cumplía con los criterios y otro manifestó no tener intención de participar en la investigación, por lo cual finalmente fueron seleccionados cuatro profesores. En segunda instancia, se enviaron consentimientos informados a los rectores, docentes y estudiantes. Continuando con el proceso, se indagó a los docentes participantes sobre las herramientas didácticas que utilizan para el desarrollo de las clases. Posteriormente, se aplica la técnica e instrumento seleccionado con el propósito de describir e interpretar los procesos de enseñanza de las competencias de la educación física mediadas por las TICS. En este sentido, se observaron dos sesiones de tres docentes que desarrollaron sus procesos de enseñanza mediante clases sincrónicas. En esta investigación se optó por observación con participación pasiva ingresando a las clases de modo incognito, sin prender la cámara o audio. Por otra parte, se revisaron tres guías de una docente que no utiliza clases sincrónicas, y se analizaron seis procesos evaluativos (dos de cada guía) de las actividades que realizaron los estudiantes. El análisis de la información se realizó mediante el software Atlas ti 9. Este software facilitó la categorización, saturación de la información para describir e interpretar los resultados.

\section{Resultados}

A continuación, se describen e interpretan las herramientas tecnológicas, estrategias didácticas, y los procesos de aprendizaje de los escolares durante las clases abordadas durante el confinamiento, asimismo, las competencias desarrolladas en las mismas. Los anteriores componentes se disertan en los siguientes subcapítulos.

\subsection{Herramientas didácticas en los procesos de enseñanza de la educación física durante el confinamiento}

En la actualidad las herramientas y estrategias didácticas de la educación física, al igual que en otras áreas del conocimiento, se transforman de acuerdo con la época y las necesidades de cada población. Desde esta perspectiva, los docentes a causa del Covid 19 se adaptaron a 
nuevas dinámicas de enseñanza, entre estas, las clases sincrónicas mediante plataformas como, por ejemplo, Microsoft Teams, Zoom meeting o Google Meet, Por otra parte, las clases asincrónicas, guías físicas o enviadas mediante correo, inclusive por WhatsApp, sesiones por televisión, tutoriales, videos de clases por WhatsApp y YouTube las cuales no generan una interacción directa, pero también son herramientas utilizadas por los educadores durante el confinamiento y fuera de este.

Inicialmente, en la presente investigación codificamos las observaciones de clases y docentes. Posteriormente, se describen las herramientas tecnológicas utilizadas en cada sesión. Finalmente, se clasificaron las fases de la clase (inicial, central y final). Lo anterior, con el propósito de describir e interpretar en detalle el proceso de enseñanza y aprendizaje escolar de los participantes desarrollados mediante las herramientas didácticas TICS.

Tabla 1. Observaciones de clases

\begin{tabular}{|l|l|l|l|l|l|}
\hline Observaciones & Código & $\begin{array}{l}\text { Institución } \\
\text { Educativa }\end{array}$ & $\begin{array}{l}\text { No } \\
\text { participantes }\end{array}$ & Plataforma & Docente \\
\hline Observación 1 & OQ1 & Colegio 1 & 21 & Meet & DQ1 \\
\hline Observación 2 & OQ2 & Colegio 1 & 18 & Meet & DQ1 \\
\hline Observación 3 & OP3 & Colegio 2 & 24 & Meet & DP2 \\
\hline Observación 4 & OP4 & Colegio 2 & 24 & Meet & DP2 \\
\hline Observación 5 & OC5 & Colegio 3 & NP & $\begin{array}{l}\text { Guías } \\
\text { enviadas por } \\
\text { correo y } \\
\text { WhatsApp }\end{array}$ & \\
\hline Observación 6 & OC6 & Colegio 3 & NP & $\begin{array}{l}\text { Guías } \\
\text { enviadas por } \\
\text { correo y } \\
\text { WhatsApp }\end{array}$ & DC3 \\
\hline Observación 7 & OC7 & Colegio 3 & NP & $\begin{array}{l}\text { Guías } \\
\text { enviadas por }\end{array}$ & DC3 \\
\hline
\end{tabular}




\begin{tabular}{|l|l|l|l|l|l|}
\hline & & & & $\begin{array}{l}\text { correo y } \\
\text { WhatsApp }\end{array}$ & \\
\hline Observación 8 & OJ8 & Colegio 4 & 15 & Teems & DC3 \\
\hline Observación 9 & OJ9 & Colegio 4 & 10 & Teems & DJ4 \\
\hline & & & & & \\
\hline
\end{tabular}

Fuente: Autoría propia

Con respecto a las herramientas didácticas, inferimos que, la herramienta didáctica más utilizada por los docentes durante las clases observadas fueron las clases sincrónicas. Las clases sincrónicas consisten en la interacción entre docentes y estudiantes a través de dispositivos electrónicos como el computador, Tablet o celulares mediante el internet. En este aspecto, hallamos un docente que utilizó la plataforma Microsoft Teams, ya que, esta es de acceso gratuito para los docentes que pertenecen a la secretaria de educación de Bogotá. Por otro lado, dos docentes trabajaron con Google Meet, el profesor DQ1 usa la plataforma de acceso gratuito y el docente DP3 utiliza la plataforma pagada por su institución educativa, la cual le permite aprovechar aplicaciones como grabar las clases entre otras. En cuanto, al promedio de estudiantes participantes de las clases sincrónicas fue de 19 estudiantes, cursos unificados. Es necesario aclarar que, normalmente un curso de quinto o cuarto en el sector oficial, oscila entre 30 y 40 estudiantes. Desde esta perspectiva, se evidencia un porcentaje muy bajo de estudiantes que desarrollan las clases mediante esta alternativa tecnológica, lo cual muestra una posible brecha digital, desigualdad social y educativa.

\subsection{Estrategias didácticas en los procesos de enseñanza para el desarrollo de las competencias de la educación física}

La clase de educación física en Colombia se enfatiza en el desarrollo de las competencias motriz, axiológico corporal, expresivo corporal y especificas en relación con las básicas. En este contexto, los docentes utilizan como ejes temáticos la enseñanza deportiva, juegos, ejercicios físicos, actividades lúdicas, la danza y recreación entre otras, los anteriores abordados mediante estrategias didácticas. A continuación, se exponen las estrategias 
didácticas utilizadas por los docentes de la presente investigación, las cuales pueden permitir el desarrollo de las competencias mencionadas en los escolares.

Tabla 2. Actividades de la fase inicial.

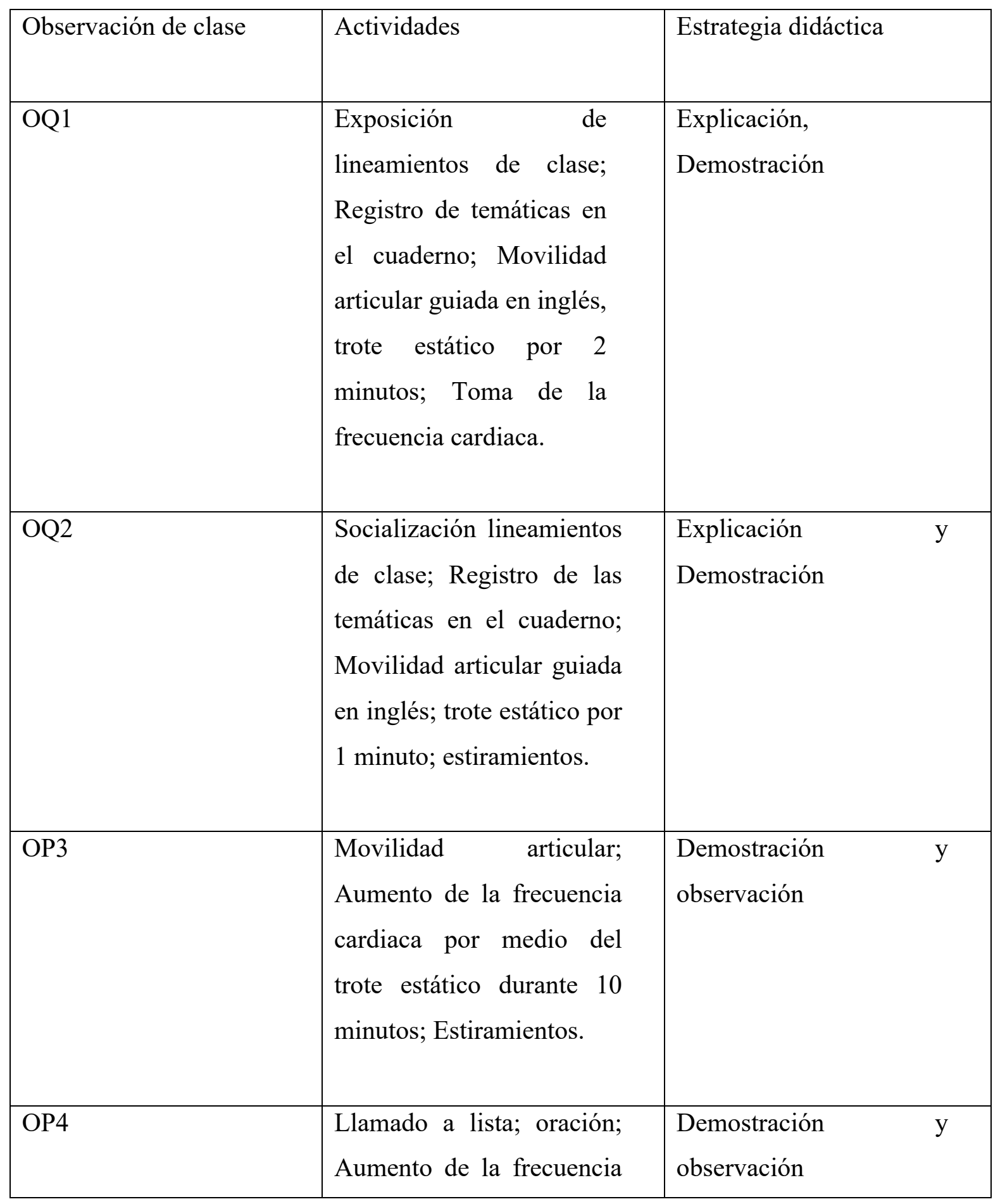




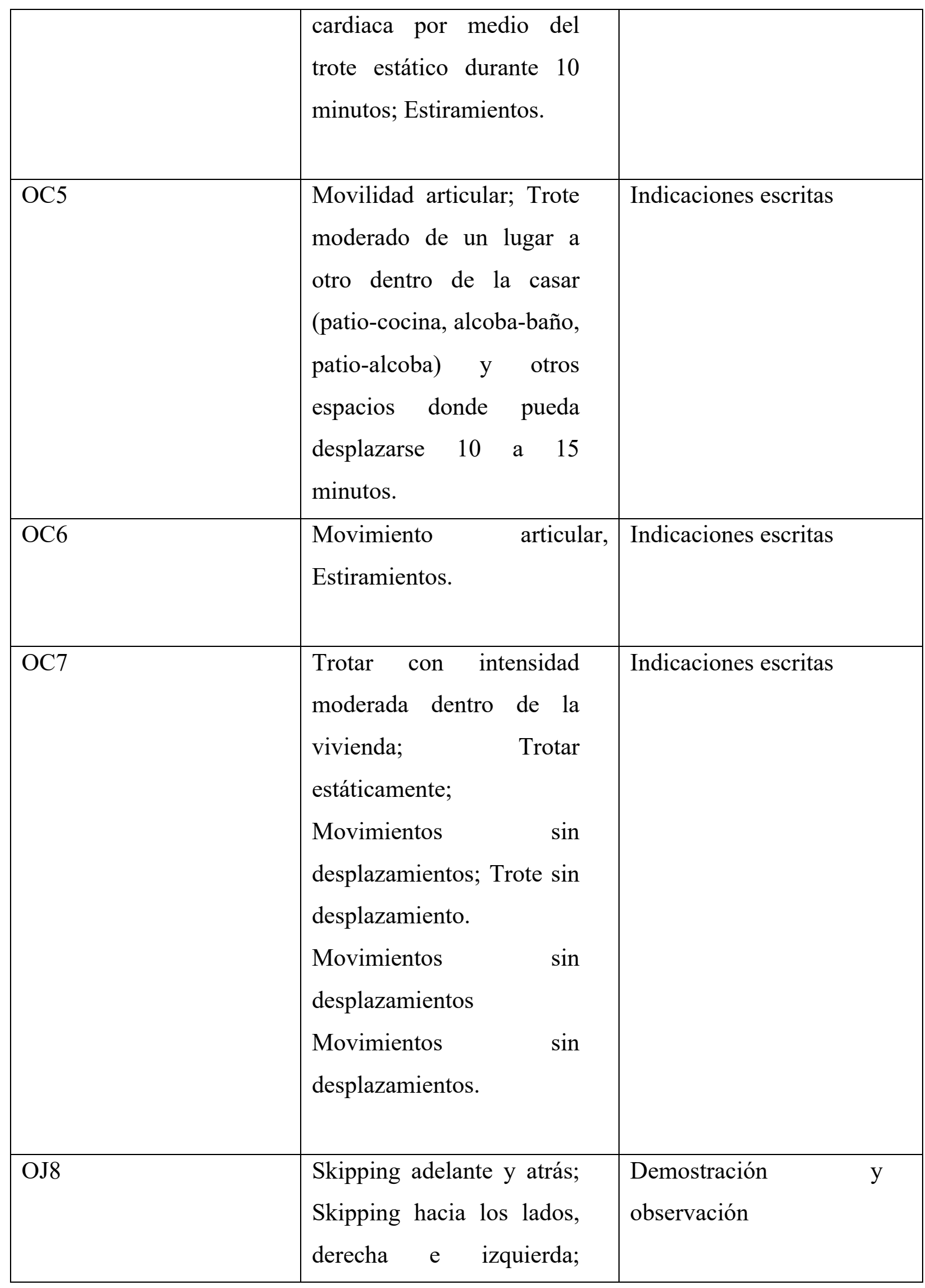




\begin{tabular}{|l|l|l|}
\hline & $\begin{array}{l}\text { Skipping con elevación de } \\
\text { pie derecho e izquierdo; } \\
\text { Skipping con elevación de } \\
\text { pie lateral derecho e } \\
\text { izquierdo. Skipping con } \\
\text { elevación de pie hacia } \\
\text { atrás; Skipping con } \\
\text { movimiento de brazos en } \\
\text { circulo hacia adelante; } \\
\text { Skipping con movimiento } \\
\text { de brazos en circulo hacia } \\
\text { atrás; Skipping con } \\
\text { estiramientos de piernas; } \\
\text { Skipping con giro del tren } \\
\text { superior. }\end{array}$ \\
$\begin{array}{l}\text { Trote suave durante 10 } \\
\text { minutos; multisaltos; } \\
\text { estiramientos. Demostración y observación }\end{array}$ \\
\hline
\end{tabular} \mid

Fuente: Autoría propia

En el presente estudio, se evidencia en la fase inicial, el desarrollo de la competencia motriz, en esta la condición física con ejercidos físicos dirigidos. En cuanto a las estrategias de didácticas, el docente DQ1 expone los lineamientos de la clase antes de iniciar con el calentamiento, además de realizar los ejercicios con sus estudiantes. Por su parte, el docente DJ4 expone los ejercicios de forma práctica, y posteriormente apaga la pantalla para observar a los estudiantes realizando las correcciones permitentes. El docente DP2, además de proponer y mostrar los ejercicios de forma práctica permanece con su cámara abierta, también observa 
y corrige a los estudiantes. A su vez, la docente DC3 envía guías de trabajo por medio del correo electrónico y el WhatsApp.

Ahora veamos, la descripción e interpretación de las observaciones en la fase central. Para ilustrar mejor, la fase central generalmente son los momentos donde se desarrollan las temáticas más importantes de la sesión de clase.

Tabla 3. Actividades fase central.

\begin{tabular}{|c|c|c|}
\hline $\begin{array}{l}\text { Observación de } \\
\text { clase }\end{array}$ & Actividades & Estrategia de enseñanza \\
\hline OQ1 & $\begin{array}{l}\text { Sentadillas; Toca talones; Elevación } \\
\text { de la rodilla derecha y la toca con la } \\
\text { mano izquierda, luego, al contrario; } \\
\text { Saltar; Dibujo del cuerpo con } \\
\text { nombre, peso, estatura, cuanto mide } \\
\text { el perímetro de la cintura, fecha de } \\
\text { nacimiento y edad }\end{array}$ & Demostración \\
\hline OQ2 & $\begin{array}{l}\text { Carrera estática; Multisaltos; } \\
\text { Lanzamientos de pelota y flexiones }\end{array}$ & Demostración \\
\hline OP3 & $\begin{array}{l}\text { Trote de } 3 \text { minutos; Sentadillas; Trote } \\
12 \text { minutos; Sentadillas con manos } \\
\text { en la cintura; Sentadillas con brazos } \\
\text { al frente; Repeticiones; Sentadillas } \\
\text { con brazos a los lados; Sentadillas } \\
\text { con manos en la cintura; } \\
\text { Abdominales; Flexiones de brazos }\end{array}$ & $\begin{array}{l}\text { Demostración } \\
\text { observación }\end{array}$ \\
\hline
\end{tabular}




\begin{tabular}{|c|c|c|c|}
\hline OP4 & $\begin{array}{l}\text { Tijeras; Saltos uniendo y separando } \\
\text { los pies, llevando brazos arriba y } \\
\text { abajo; Saltos llevando pie derecho } \\
\text { adelante y brazo izquierdo arriba, } \\
\text { posteriormente cambiar la posición; } \\
\text { Carrera continua de forma; Subir y } \\
\text { bajar brazos; brazos al frente, arriba y } \\
\text { abajo; brazo derecho arriba e } \\
\text { izquierdo abajo y alternar; Trotar } 2 \\
\text { minutos; Caminar a paso largo; } \\
\text { Trotar } 3 \text { minutos; Descansar; Trotar } 5 \\
\text { minutos; Descansar, beber agua y } \\
\text { comer bocadillo; Trotar } 7 \text { minutos }\end{array}$ & $\begin{array}{l}\text { Demostración } \\
\text { observación }\end{array}$ & \\
\hline OC5 & $\begin{array}{l}\text { El estudiante debe ubicarse en la } \\
\text { puerta de acceso de su hogar, debe } \\
\text { trasladarse a uno de los puntos del } \\
\text { hogar y obtener algún objeto, } \\
\text { simulando como si lo estuviera } \\
\text { comprando en el supermercado, } \\
\text { llevándolo hacia la puerta donde } \\
\text { comenzó y dejarlo. Este ejercicio se } \\
\text { debe realizar de la puerta-baño, luego } \\
\text { puerta-cuarto, seguido puerta-cocina } \\
\text { y por último puerta-patio o la azotea; } \\
\text { Sumados todos los sitios recorridos } \\
\text { se le llama un circuito. }\end{array}$ & Indicaciones escritas & \\
\hline OC6 & $\begin{array}{l}\text { Dibuja la figura geométrica que más } \\
\text { te guste, de } 30 \mathrm{~cm} \text { de ancho } \\
\text { aproximadamente, y recórtala. }\end{array}$ & Indicaciones escritas & \\
\hline
\end{tabular}




\begin{tabular}{|c|c|c|}
\hline & $\begin{array}{l}\text { Consigue una pelota que bote. Pega } \\
\text { tu figura en una pared e intenta } \\
\text { golpearla con la pelota muchas veces. } \\
\text { Intenta lanzarla de varias formas o } \\
\text { golpearla con diferentes partes del } \\
\text { cuerpo. Cuando haya rebotado en la } \\
\text { figura, deja que bote una vez en el } \\
\text { suelo y trata de atraparla. }\end{array}$ & \\
\hline OC7 & $\begin{array}{l}\text { En este reto emplearás la fuerza y la } \\
\text { velocidad para resolver situaciones } \\
\text { de juego. Además, identificarás en } \\
\text { qué momento es necesario anticiparte } \\
\text { y oponerte a los movimientos de tus } \\
\text { adversarios. } \\
\text { Uno de los participantes se colocará } \\
\text { acostado boca abajo, con las piernas } \\
\text { y brazos extendidos y separados. El } \\
\text { jugador que se encuentra de pie } \\
\text { intentará, utilizando su fuerza, voltear } \\
\text { a su adversario en el menor tiempo } \\
\text { posible. El otro jugador tratará de } \\
\text { evitar que lo volteen. Una vez que el } \\
\text { jugador que está de pie logre voltear } \\
\text { a su adversario, podrán cambiar los } \\
\text { papeles y jugar de nuevo. Invita a } \\
\text { otras personas para formar dos } \\
\text { equipos y mientras la mitad se pone } \\
\text { boca abajo, la otra mitad intenta }\end{array}$ & Indicaciones escritas \\
\hline
\end{tabular}




\begin{tabular}{|l|l|l|}
\hline & $\begin{array}{l}\text { voltearlos. Traten de hacerlo en } \\
\text { menos tiempo que el adversario, } \\
\text { analizando aspectos importantes del } \\
\text { juego como la anticipación a los } \\
\text { movimientos del otro y la oposición } \\
\text { oportuna a la fuerza o dirección de los } \\
\text { movimientos del adversario. }\end{array}$ & y \\
\hline OJ8 & $\begin{array}{l}\text { Flexiones de brazos; Salto lateral con } \\
\text { apoyo de un pie; Multisaltos con } \\
\text { flexión profunda; Salto vertical }\end{array}$ & Demostración \\
observación \\
\hline OJ9 & $\begin{array}{l}\text { Salto sobre una silla; Saltos a pie } \\
\text { juntos; trote con flexión de brazos. }\end{array}$ & Demostración \\
& observación \\
\hline
\end{tabular}

Fuente: Autoría propia

Con respecto, a las actividades propuestas en la fase central, encontramos que la tendencia es el desarrollo de la competencia motriz, con énfasis en la condición física a través del ejercicio físico. En esta fase, se destaca el docente DQ1 en la OQ1 porque relaciona algunas actividades con situaciones que se pueden presentar en la vida diaria, estimulando la comprensión para el desarrollo de la competencia motriz. (min 35): Entonces usted cuando juega baloncesto tiene que saltar, usted cuando juega voleibol tiene que agacharse y saltar y bloquear y todas estas posturas nos sirven para nuestra vida. Resulta que me voy a ir en el bus y se me cae la moneda, tengo que agacharme, pero si no hago los ejercicios me duele la espalda, me lanzan algo me puedo agachar. Todas estas posturas nos sirven para la cotidianidad (OQ1).

Asimismo, la docente DC3 en algunas las guías proponen actividades relacionando la vida cotidiana. En este aspecto en la OC5, fase central de la guía, la docente propone: "El estudiante debe ubicarse en la puerta de acceso de su hogar, debe trasladarse a uno de los puntos del hogar y obtener algún objeto, simulando como si lo estuviera comprando en el supermercado, llevándolo hacia la puerta donde comenzó y dejarlo”. 
Lo anterior permite desarrollar la comprensión, y de esta manera la competencia motriz. Según. Perkins (1995) “El aprendizaje es una consecuencia del pensamiento. Sólo es posible retener, comprender y usar activamente el conocimiento mediante experiencias de aprendizaje en las que los alumnos reflexionen sobre lo que están aprendiendo y con lo que están aprendiendo" (p.21) En este sentido, observamos algunos elementos que permiten la relación de las actividades con la vida cotidiana. Sin embargo, la tendencia en la fase central son los ejercicios físicos guiados sin reflexión o explicación del para qué.

\subsection{Procesos de aprendizaje en el desarrollo de las competencias de la educación física mediante las clases sincrónicas}

En el presente estudio, como se expuso en epígrafes anteriores, se hallan las clases sincrónicas como la herramienta didáctica más utilizada por los docentes. Estas permiten observar los procesos de aprendizaje de los escolares, actuaciones y comportamientos. Sin embargo, los dispositivos electrónicos también pueden limitar la observación, procesos de enseñanza y aprendizaje cuando los estudiantes deciden no prender su pantalla, o se presentan problemas de conexión. En particular, los procesos de aprendizaje desarrollados mediante las clases sincrónicas evidencian la necesita de una constante interacción entre docente y estudiante a través de los dispositivos tecnológicos. Ahora bien, en este estudio se observaron algunas situaciones que permiten inferir las dificultades que presentan los procesos de enseñanza y aprendizaje durante las clases sincrónicas.

En primera instancia, describimos actuaciones y comportamientos de los estudiantes en la fase inicial, las cuales no favorecen la comprensión de las temáticas propuestas. Llegado a este punto, en la sesión de clase OP3 (minuto 4) "Los estudiantes siguen las actividades que propone el docente, algunos permanecen con la cámara apagada, por lo cual, no se evidencia el desarrollo de sus actividades.” Por otra parte, en la observación de clase OP4 (minuto 8) "El estudiante llamado Juan (para ocultar su identidad), se encuentra en un sitio rural, aunque la cámara lo enfoca no parece prestar atención a las indicaciones del profesor, 
después de 3 minutos se acerca a la pantalla, luego se retira por una silla, la lleva cerca de la cámara y se sienta para prestar atención.” (...) "En el caso de Maicol (nombre asignado para ocultar su identidad), se observa la cámara desactivada. Sin embargo, al activarse la cámara se evidencia que no se encuentra en la clase. Por otro lado, Manuel se encuentra con la acudiente escuchando el llamado a lista del docente”.

El docente DP2, antes de iniciar las actividades correspondientes al desarrollo de las competencias de la educación física realizó una oración, durante esta actividad se observó que varios niños apagan la cámara, otros prestan atención, y algunos realizan movimientos como caminar, sentarse y adaptar la posición de pie entre otros. Finalmente, en esta fase cuando el profesor inicia el calentamiento (OP4) (minuto 14) Maicol se desplaza hacia la puerta de la habitación, allí se encuentra con otro niño, con el cual inicia un dialogo, se acerca al equipo y apaga la cámara (OP4). En las clases de los otros docentes participantes del estudio, no se observan actuaciones particulares en los estudiantes. No obstante, varios estudiantes permanecen con la cámara apagada, por lo cual no se pueden observar sus actuaciones y comportamientos.

Continuando con la descripción, en la fase central, hallamos las siguientes actuaciones y comportamientos de los estudiantes: En la observación de clase OQ1 (minuto 16) se observa a Juan David, que solo se encuentra observando la pantalla. En el (minuto 17) Martin continua de pie, camina, se aleja de la pantalla, abre la puerta y sale del cuarto. Con respecto a la observación OP4 (minuto 36) se presentan acciones que se distancian de las clases como, por ejemplo, "se observa a julio quien constantemente manipula una bomba pequeña, esta actividad no le permite realizar el ejercicio como lo propone el docente” Asimismo, "Algunos niños en la actividad de carrera continua de 3 minutos, se acercan a la cámara y simulan trotar, otros se detienen continuamente y solo 6 niños realizan la actividad propuesta" Por su parte "Robin pregunta si puede tomar agua. Rocío juega con su gatico y hermana en el tiempo de descanso". 
En consonancia, la OP3 (minuto 11) evidencia en el ejercicio de carrera continua, que algunos estudiantes apagan la cámara. Por su parte, Jorge se detiene y pasados 30 segundos continua con el trote. Luis se acerca a la cámara para preguntar algo, él no se encuentra realizando la carrera continua. En el caso de la OJ8 (minuto 13) Después de la explicación del siguiente ejercicio, "salto hacia adelante," 11 estudiantes prenden sus cámaras e inician a hacer las actividades, 6 no prenden su cámara. Sin embargo, cuando el docente los nombra por este hecho 4 activan su cámara.

En las anteriores actuaciones y comportamientos de los estudiantes nos permite inferir que, los estudiantes al presentar poca motivación deciden realizar actividades diferentes a las propuestas en clase, interrumpiendo su proceso de aprendizaje. Cabe señalar que, el uso de los dispositivos les permite manipular la interacción, asistir a la clase, pero no participar activamente de ella. Por otra parte, las clases sincrónicas no permiten interpretar las acciones de los escolares cuando estos no prenden su cámara, algunos por problemas de conectividad, y otros probablemente no les interesa participar. Lo anterior, nos muestra un panorama un poco complejo teniendo en cuenta que, los docentes necesitan observar constantemente a sus estudiantes para lograr guiar de una mejor manera los procesos de aprendizaje escolar.

Ahora bien, los escolares que participan activamente demostraron las siguientes actuaciones y comportamientos. Inicialmente, exponemos la OQ1 "Los estudiantes en esta fase prestan atención a los argumentos del profesor, y realizan preguntas cuando no entienden alguna explicación sobre los retos de la semana." Estos retos consisten en tareas motrices que los estudiantes deben practicar durante la semana y presentar en el siguiente encuentro sincrónico. En cuanto a la clase OJ8 "los estudiantes preguntan cuándo no entienden. Con respecto al ejercicio propuesto por el docente (salto lateral con apoyo de un pie) lo ejecutan siguiendo el ejemplo del docente. Cuando terminan las series le informan al profesor y apagan las cámaras por indicación del docente. Por otra parte, la docente DC3 no realiza clases sincrónicas, ella propone guías, las cuales envía mediante el WhatsApp y correo electrónico. El proceso de aprendizaje escolar debe ser autónomo o guiado por los acudientes. La evidencia del trabajo de los escolares es mediante envío de videos cortos, aproximadamente 3 minutos 
mostrando la actividad central. Con respecto a la fase final, no se evidencia con claridad, generalmente en este espacio los docentes motivan y promueven a los estudiantes para practicar actividad física en casa, se despiden de los estudiantes y aquellos que prendieron su cámara la apagan.

\section{Discusión}

En Colombia los procesos de enseñanza en la educación física se enfatizan en el desarrollo de las competencias propuestas por las orientaciones pedagógicas del año 2010. Ahora bien, las clases de educación física mediadas por las Tics durante el confinamiento pueden facilitar el desarrollo de estas competencias. No obstante, en las observaciones realizadas, se evidencia una tendencia en el abordaje de la competencia motriz, específicamente en el desarrollo de la condición física mediante los ejercicios físicos dirigidos desarrollando muy poco las demás competencias. En consonancia, Baena et al (2021) quienes consideran que la enseñanza de la educación física $(\mathrm{EF})$ durante la pandemia se enfatiza en la preservación de la salud física y mental, demostrando como prioridad la enseñanza de la condición física y la salud. Con respecto a la competencia expresivo corporal y motriz a través de la enseñanza de técnicas deportivas y habilidades motoras pasan a un segundo plano. Por su parte, San Frutos (2021) manifiesta que, la EF durante el confinamiento permitió el bienestar de los estudiantes. Sin embargo, muchos educandos quedaron por fuera del sistema educativo evidenciando desigualdad social y educativa.

Con respecto a las herramientas didácticas utilizadas por los docentes, encontramos las clases sincrónicas como la alternativa más utilizada en los procesos de enseñanza y aprendizaje durante el confinamiento a causa del Covid 19. Esta herramienta puede permitir la interacción entre estudiantes y docente para desarrollar procesos de enseñanza y aprendizaje a través de dispositivos, como, el celular y computador. No obstante, se encuentran varias limitaciones en cuanto a la interacción y acceso de todos los estudiantes. En particular, hallamos estudiantes que no interactúan o participan de las clases por diferentes motivos, entre estos posiblemente por la falta de recursos tecnológicos, motivación o significancia de la clase. Lo 
anterior debe ser un elemento de constante reflexión docente, ya que, los dispositivos muestran una realidad educativa generando un desafío en los procesos de enseñanza y aprendizaje escolar. En consonancia, Cañizales et al (2021) consideran las clases sincrónicas son un desafío para los profesores, porque necesitan de la creatividad con el propósito que el estudiante logre una significancia de forma. Dicho lo anterior, la participación del escolar en gran parte depende de las estrategias didácticas que implemente el docente y el uso adecuado de los dispositivos digitales.

Cabe señalar que, las clases sincrónicas generalmente se realizan desde casa o sitios cerrados mediante plataformas. En este caso, las plataformas utilizadas por los docentes del presente estudio fueron: Microsoft Meet y Google Meet, las cuales permiten la interacción directa entre dos o más personas a través de dispositivos como, el celular, Tablet y computador. También hallamos una docente que realiza sus procesos de enseñanza mediante el correo electrónico y WhatsApp, este último facilita la comunicación oportuna mediante audios, videos y llamadas los cuales se pueden observar en diferentes horas y varias veces. En contraste, Escobar et al. (2018) quienes analizaron las experiencias sobre la utilización del video juego como estrategia didáctica. En esta investigación, los autores consideran que el uso de los videojuegos estimula la resolución de problemas en el aprendizaje, desarrolla habilidades cognitivas y motrices, además de mejorar la creatividad. Por su parte, Azócar (2021) implementó los videojuegos como la herramienta didáctica utilizada durante la pandemia por Covid 19. El objetivo fue aumentar la actividad física en los escolares, ya que, estos no necesitan de salir de casa, además de ser una alternativa que motiva a la mayoría de las escolares. Ahora bien, encontramos limitaciones en los videojuegos, como la interacción entre docente y estudiantes sino deciden jugar en línea, pero facilita la integración entre miembros del hogar o amigos.

Con respecto a las estrategias didácticas algunas plataformas permiten crear salas, reuniones de pequeños grupos promoviendo el trabajo colaborativo, la interacción, el trabajo en equipo, inclusive la exploración. En otras palabras, el docente debe ser creativo y aprovechar al máximo las herramientas, además de aplicar las estrategias didácticas que faciliten la comprensión para desarrollar las competencias del área. Desde esta perspectiva, los docentes 
del presente estudio utilizan las herramientas didácticas, pero sus estrategias didácticas son basadas en el trabajo individual y autónomo de los escolares limitando las posibilidades que brindan las clases sincrónicas. En concordancia, con González et al (2021) quienes en su investigación exponen la virtualidad con deficiencias, además los decentes aumenta la probabilidad de sufrir estrés docente y desagrado. También concluyen que, los futuros docentes manifiestan la enseñanza de la EF presencial como aspecto indispensable para no perder su esencia.

Desde otro punto de vista, en la actualidad el sistema educativo propone modelos didácticos que se asocian con postulados constructivistas, como, por ejemplo, el aprendizaje cooperativo de Johnson y Johnson, en el cual los escolares trabajan juntos para lograr un objetivo común (Johnson y Johnson, 1999) En concordancia, Carter et al. (2018) manifiestan, que las actividades cooperativas permiten la interacción social y el aprendizaje motriz, además el deporte puede ser el principal motivo de los escolares para asistir a la escuela aumentando el capital social. Por otra parte, el aprendizaje por descubrimiento, en este Castillo et al (2020) consideran que, el aprendizaje por descubrimiento produce estudios con relevancia, puesto que los temas propuestos en el plan de estudios deben ser descubiertos por el estudiante generando un proceso de aprendizaje activo. En contraste con lo anterior, los profesores participantes en el presente estudio desarrollan sus procesos de enseñanza a través de la demostración y explicación de los ejercicios propuestos. Por su parte, los escolares imitan los movimientos y actividades desarrollando sus capacidades físicas de manera individual. En el caso de las guías, los estudiantes siguen las indicaciones de estas en forma autónoma o guiados por los acudientes, pero con poca comprobación. Lo anterior puede generar unos procesos de enseñanza poco comprensivos, porque limita la exploración, construcción de aprendizajes y la interacción motriz, pero mejora puede desarrollar la condición física, la disciplina y autonomía.

Finalmente, consideramos que las Tics pueden evidenciar los aprendizajes comprensivos a través de las acciones y comportamientos de los escolares durante la sesión de clases. En este sentido, las actuaciones y comportamientos evidencian el proceso de aprendizaje de los 
estudiantes. En la presente investigación se muestran tres perspectivas. La primera, son los procesos de aprendizaje que realizan los escolares siguiendo los ejercicios con la pantalla prendida, indagando y mostrando motivación hacia la clase. La segunda, escolares que a pesar de prender su cámara realizan actividades diferentes a las propuestas durante la clase. Y la tercera, los estudiantes que prenden ocasionalmente la pantalla o no lo hacen generando poca comprobación del desarrollo de las actividades. Las dos últimas perspectivas demuestran poca motivación de los escolares hacia la clase. En este aspecto Sánchez et al (2014) consideran la motivación como una de las necesidades psicológicas, las cuales inciden en comportamientos positivos. También facilitan los comportamientos de adaptación en los contextos de la educación física. De manera semejante, Marín de Oliveira. (2016) confirma el protagonismo de la motivación en las decisiones y comportamientos positivos de los escolares frente a los procesos de aprendizaje, además la baja de comportamientos de indisciplina. En síntesis, inferimos que posiblemente los escolares no prenden su pantalla por la falta de motivación o adaptación al nuevo contexto educativo.

\section{Conclusiones}

Los procesos de enseñanza y aprendizaje escolar necesitan de las herramientas y estrategias didácticas para tratar de lograr la comprensión en los estudiantes, y de esta manera adquirir competencias necesarias para interactuar en el contexto. No obstante, el virus Covid 19 causó transformaciones en los procesos de enseñanza y aprendizaje escolar a nivel mundial, en este panorama los docentes y sistemas educativos dejaron a un lado estrategias y herramientas didácticas que eran utilizadas frecuentemente para adoptar herramientas que presentaban poca tendencia, como es el caso de las TICS. Cabe señalar que, las Tics exponen diferentes herramientas didácticas, entre estas las clases sincrónicas son las que permiten mayor interacción entre estudiantes y docente, pero no todos los estudiantes tienen acceso a esta herramienta didáctica. La dificultad de conexión de varios estudiantes y docentes generan la necesidad de acceder a otras herramientas, como, por ejemplo, classroom, sesiones de clases por YouTube, videos y guías enviadas a través del correo electrónico WhatsApp entre otras estrategias. 
En el presente estudio se evidencian las clases sincrónicas como la tendencia en el desarrollo de las clases durante el confinamiento escolar. Las clases sincrónicas pueden permitir desarrollar estrategias didácticas según el saber e intención del docente. Sin embargo, los profesores hacen hincapié en la explicación y demostración de ejercicios físicos para desarrollar la condición física, la cual hace parte de la competencia motriz siendo el principal objetivo de las clases observadas. Lo anterior, se relaciona con el proceso de aprendizaje escolar, ya que, la mayoría de los estudiantes imitan, siguen las instrucciones de los docentes, y en alagunas ocasiones realizan preguntas cuando no entienden un ejercicio propuesto. Por otra parte, los estudiantes que no presentan motivación, agrado o significancia sobre la clase no prenden su cámara o lo hacen ocasionalmente, y algunos realizan otras actividades lo cual dificulta su seguimiento motriz.

Con respecto a las competencias de la EF desarrolladas mediante las TICS durante el confinamiento, hallamos que poco se abordan la competencia expresivo corporal, axiológico corporal y especificas en relación con las básicas. Lo anterior, genera limitaciones en los propósitos de enseñanza planteados en las orientaciones pedagógicas del año 2010. Ahora bien, aunque en la actualidad varias instituciones reabrieron sus puertas para reestablecer la formación presencial, muchos estudiantes no regresaron por temor a posibles contagios, esta situación obliga a los docentes continuar usando algunas herramientas didácticas tecnológicas, por lo cual inferimos que seguirán siendo fundamentales en los procesos de enseñanza escolar. Dice González et a 1 (2021) que los docentes necesitan formación pedagógica en TICS, además de sugerencias para el retorno presencial. A manera de síntesis, consideramos las TICS como herramientas didácticas necesarias en el confinamiento, pero debe ser tenida en cuenta en la reapertura total de la escuela, como un complemento educativo. En consonancia, los docentes deben capacitarse constantemente en el uso de la tecnología, ya que, esta presenta muchas posibilidades para desarrollar las competencias de la educación física. 


\section{Referencias}

Álvarez, C. (2008) La etnografía como modelo como modelo de investigación en educación. Gazeta de Antropología.

Azócar-Gallardo, A. Ojeda-Aravena. (2021) Los videojuegos activos en tiempos de pandemia por COVID-19: una potencial estrategia para aumentar la actividad física de los escolares, Fisioterapia, Volumen 43, Issue 2, 2021, Page 124, ISSN 0211-5638, https://doi.org/10.1016/j.ft.2021.01.001

Baena Morales, S., López Morales, J., y García-Taibo, O. (2021). La intervención docente en educación física durante el periodo de cuarentena por COVID-19. Retos: Nuevas Perspectivas de Educación Física, Deporte y Recreación, 39, 388-395.

Barahona, J., Molina-García, J., y Monfort-Pañego, M. (2020). Physical education teachers' knowledge and educational intentionality in the use of ICT. [El conocimiento y la intencionalidad didáctica en el uso de TIC del profesorado de educación física] Retos, 83, 497-504.

Cañizares Galarza, F. P., Quevedo Arnaiz, N. V., \& García Arias, N. (2021). Retos de la enseñanza-aprendizaje virtual: creatividad del docente, clases sincrónicas o asincrónicas, y principios didácticos. Revista Conrado, 17(S1), 331-339.

Castillo Rodríguez, N. J., Giraldo Santamaría, D., y Zapata-Gordon, A. (2020). Aprendizaje por Descubrimiento: Método Alternativo en la Enseñanza de la Física. Scientia et Technica, 25(4), 569-575. https://doiorg.ez.urosario.edu.co/10.22517/23447214.24221

Carter-Thuillier, B., López-Pastor, V y Gallardo-Fuentes. (2018). Enseñanza para la comprensión y deporte escolar: un estudio en contexto intercultural y situación de riesgo social. Infancia y Aprendizaje. 
Ciotti, M., Ciccozzi, M., Terrinoni, A., Jiang, W. C., Wang, C. B., \& Bernardini, S. (2020). The COVID-19 pandemic. Critical reviews in clinical laboratory sciences, 57(6), 365-388.

De Camelloni, A. (2008). El saber didáctico. Editorial: Paidós

Diaz Barriga, A. (2009). Pensar la didáctica. Amorrortu editores

Escobar, M., Sanhueza, Susan y Friz, Miguel (2018). Uso de estrategias tecnológicas en educación: una comparación entre biología y educación física. Revista Mexicana de Investigación Educativa, 23(77),483-504.

Gardner, H. (2016) La mente no escolarizada. Cómo piensan los niños y cómo deberían enseñar las escuelas. Editorial. Paidós.

González-Calvo G, Barba-Martín RA, Bores-García D, Hortigüela-Alcalá D. The (virtual) teaching of physical education in times of pandemic. European Physical Education (2021) doi:10.1177/1356336X211031533

González-Rivas, R. A., Gastélum-Cuadras, G., Velducea, W. V., Bustos, J. B. G., \& Esparza, S. D. (2021). Analysis of teaching experience in physical education classes during COVID19 confinement in mexico. [Análisis de la experiencia docente en clases de Educación Física durante el confinamiento por COVID-19 en México] Retos, 42, 1-11. doi:10.47197/RETOS.V42I0.86242

Johnson y Johnson (1999) Aprender Juntos y solos. Editorial Aique

Kamoga, S y Varea, V. (2021). 'Let them do PE!' The 'becoming' of Swedish physical education in the age of COVID-19. European Physical Education Review. https://doi.org/10.1177/1356336X211036574 
Ley General de Educación. Ley 115 de 1994, Constitución Política de Colombia.

Limas, V. S. (2000). La didáctica, el constructivismo y su aplicación en el aula.

Marín de Oliveira, L. (2016). Influencia de la elección de tareas y la participación del alumno en la motivación, coeducación y comportamientos disciplinados en educación física. Apunts: Educación Física y Deportes, 2(104), 138.

Maturana Moreno, G y Garzón Daza, C (2015) La etnografía en el ámbito educativo: una alternativa metodológica de investigación al servicio docente. Revista Educación y Desarrollo Social // Vol. 9 No. 2 // ISSN 2011-5318 · e-ISSN 2462-8654

Medina Rivilla, A y Mata, F, González. (2009). Didáctica general: Pearson Prentice Hall.

Perkins, D. (1995). Escuela inteligente (Vol. 17). Editorial: Gedisa. Cap. 1

Perkins, D. (1999). ¿Qué es la comprensión? La enseñanza para la comprensión, (p. 4) 69-92.

San Frutos Muñoz, L. (2021). La Educación Física en tiempos de Covid: respuestas y adaptaciones.

Sánchez Oliva, D., Viladrich, C., Amado Alonso, D., González-Ponce, I., y García Calvo, T. (2014). Predicción de los comportamientos positivos en educación física: una perspectiva desde la teoría de la autodeterminación. Revista de Psicodidáctica, 19(2), 387-406.

Secretaría de Educación (2018) (SED) Bosa localidad 7 Bogotá, D.C. Caracterización del Sector Educativo. 
Stone Wiske, M, (1999). La enseñanza para la comprension. Vinculación entre la investigación y la práctica. Editorial Paidós.

Úbeda colomer, J., y Molina Alventosa, J. P. (2016). El blog como herramienta didáctica en educación física: la percepción del alumnado. Apunts: Educacion Fisica y Deportes, (126), 37-45. https://doi-org.ez.urosario.edu.co/10.5672/apunts.2014-0983.es.(2016/4).126.04

UNESCO (2021) Interrupción y respuesta educativa. 